\title{
Preparation and Properties of Graphene Straw Retardant Composites
}

\author{
Li-guang XIAO ${ }^{\text {la, }}{ }^{*}$, Qing-shun $\mathrm{LIU}^{2, \mathrm{~b}}$ and Hong-liang $\mathrm{HU}^{3, \mathrm{c}}$ \\ ${ }^{1,2,3}$ Address:College of Material Science and Engineering, Jilin Jianzhu University, Changchun,China \\ axlg627@163.com, bqingshunliu@126.com, ,honglianghu@126.com
}

\begin{abstract}
This article was prepared by spin-coating the evaporation process graphene oxide having a shell core structure $\mathrm{GO} /$ straw flame retardant composite materials, through the oxygen index apparatus and SEM measured the relationship between the flame retardant properties and the morphological structure of the flame retardant composite material, the experiment preparation process is simple, environmentally friendly non-toxic, and the resulting $\mathrm{GO} /$ straw flame retardant composite material having a high fire retardant properties.
\end{abstract}

\section{Introduction}

China is a large agricultural country, has rich straw resources. According to the survey, China mainly deal with straw through incineration, landfill and other ways, only a small number of slices for building and plank, utilization rate is specially low ${ }^{[1]}$. Therefore, how to improve the utilization value of straw is a problem of universal attention in recent years. As we all know, graphene is a two-dimensional periodic honeycomb lattice structure consisting of six-membered carbon ring, its basic structural unit is the most stable six-membered ring of benzene of an organic material. Graphene as a kind of new graphite material, has high specific surface area and superior thermal stability ${ }^{[2]}$, etc. In this article, we prepared $\mathrm{GO} /$ straw composite materials with nuclear shell structure through rotary evaporation coating preparation, the material has good fire-retardant properties. Currently, there is no report of this research at home and abroad.

\section{Experiment Procedures}

\section{Reagent and instrument}

This experiment adopts the main reagents: Sodium nitrate, Potassium permanganate, Sulfuric acid, Hydrogen peroxide, Hydrochloric acid, nitric acid, Deionized water, purity $99.9 \%$ of graphite. Unplug the pith corn of straw, This experiment adopts the main instrument:Electrothermal blowing(WGL-65B), adjustable-speed blender(DW-I), Intelligent water bath pot(HH-2K2); Oxygen index apparatus (XYC -100 D); scanning

* Corresponding author:xlg627@163.com 
e0lectron microscope (SEM): (TM3030-CC61) and (KYKY-2800B); Sonic cleaners (WD-9415B)

\section{Experimental steps}

Unplug the pith corn of straw into the ball mill and screening of straw powder, get different particle size of straw fiber was obtained. According to Hummer ${ }^{[3]}$ method to product GO. At first, a certain amount of natural flake graphite placed in a container with concentrated sulfuric acid, stirring constantly, After mixing evenly, put the quantitative potassium permanganate and sodium nitrate into the beaker, Stirring in the ice water mixture at $0^{\circ} \mathrm{C}$, Controling reaction temperature is not more than $20 \mathrm{mins}$, and reaction heats up to $35^{\circ} \mathrm{C}$ after 30 mins, continuous stirring $1 \mathrm{~h}$ in this state. After the middle temperature reaction, the temperature rise to $80^{\circ} \mathrm{C}$, and the de ionized water was $30 \mathrm{~min}$, adding in deionized water to react $30 \mathrm{~min}$.After the completion of the high temperature reaction to add $5 \%$ of the hydrogen peroxide until the solution is bright yellow. Finally, repeatedly washed to the sulfate free with the Hydrochloric acid contain $10 \%$, GO obtained by repeated centrifugation and washed with water to neutral, then dried for 12 hours at $55{ }^{\circ} \mathrm{C}$, we got lamellar GO. Dissolved GO in water, take appropriate amount GO solution mixed with a particle size of straw not more than $0.01 \mathrm{~cm}$. After ultrasonic treatment the straw fibers are uniformly dispersed in the GO solution, then heated in the heating apparatus to evaporate, and stirred under an electric mixer continuely until the mixture reaches sticky date, the resulting sample was dried to obtain a GO / straw composites. Take the fire-retardant material in 20MPa molded to do oxygen index detector.

\section{Results and Discussion}

\subsection{GO/straw flame retardant composite material apparent characteristics analysis}

Using different content the GO mixed with particle morphology of the same straw, after ultrasonic processing straw fiber dispersed evenly in the solution. Then take it for thermostatic evaporating in the heating device, until the mixture got thicken. Dried the Sample, we get the GO/straw flame retardant composite material. As shown from fig.1

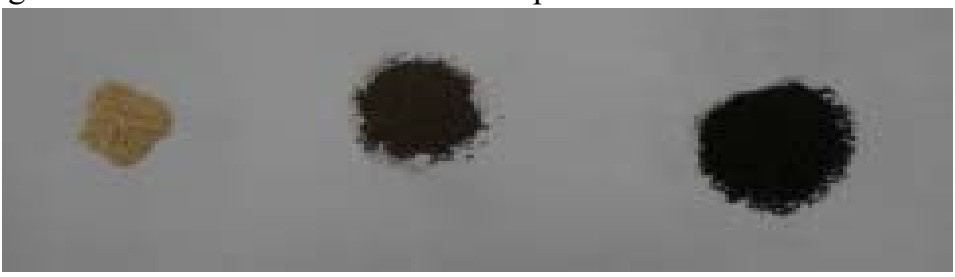

Fig.1 Different graphene oxide content of straw composites apparent figure

As you can see from Fig.1, With the increase of GO content gradually, Straw surface appears light yellow, and become brown to dark brown gradually. The phenomenon shows that, strong Vander Waals force between graphene layers, graphene can be achieved wrapped tightly in the straw powder surface, ultimately, straw powder particles surface form a dense protection carbon layer. 


\subsection{Influence of straw form on grapheme oxide's dispersion property}

In order to further exploration of the Graphene in straw particles coated on the surface of the state, the SEM observation of straw composite materials. State of straw and straw composite material micro structure images and the corresponding macro diagram is as shown from fig. 2 .
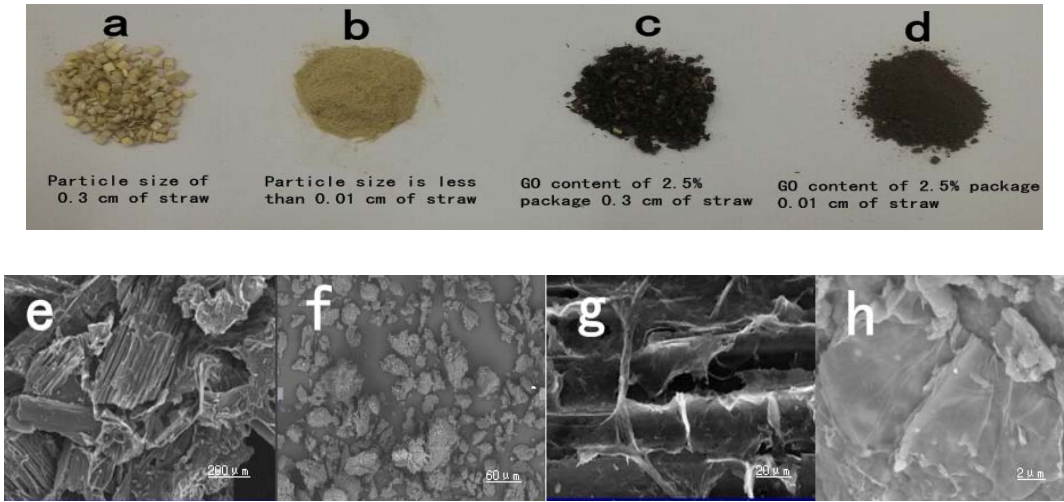

Fig.2 State of straw and straw composite material micro images and the corresponding macro structure diagram

As is shown from fig.2, different forms of straw with GO for adsorption, get the $\mathrm{GO} / \mathrm{straw}$ flame retardant composite material. Maximum diameter of $0.3 \mathrm{~cm}$ of the surface of the straw is a not fully the state of the parcel, as is shown from fig.2-c. Although the size is not more than $0.01 \mathrm{~cm}$ of straw will appear in the process of experiment phenomenon of reunion, but GO on the surface of the dispersion are better than the straw particle size of $0.3 \mathrm{~cm}$, as is shown from fig2-d. Based on the GO/straw flame retardant composite material for SEM observation, as is shown from fig2e-h. When straw particle size is larger, Straw is concave and convex shape skeleton, for which GO cannot dispersed uniformly on the surface of straw, and it appears phenomenon of fault, adhesion and multi-layer cover. After grinding process, the size of the straw particle gets smaller, specific surface area gets larger, which achieve the compact parcel of straw and GO, as is shown from fig.2-h.

The experiment makes a determination on oxygen index of different straw with same content of GO. The relation of straw composites with different oxygen index and straw particle size is shown from fig. 3 . 


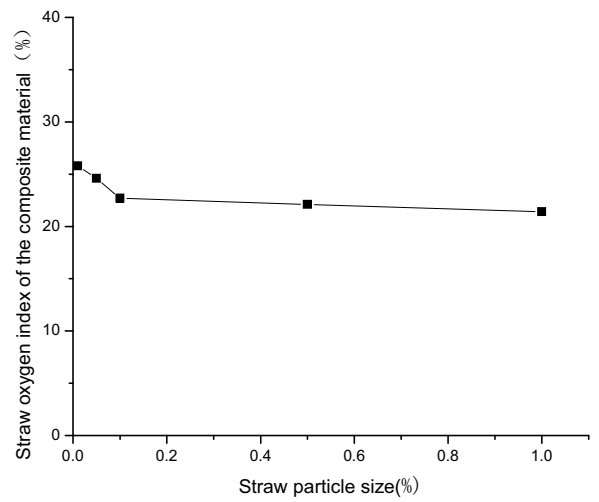

Fig.3 The relation of straw composites with different oxygen index

and straw particle size

As shown from fig.3, with the increasing of particle size of straw, the oxygen index of compound material of straw showed a downward trend, the rate of change of the oxygen index of compound material of straw was decreased, when the particle size of straw was greater than $0.05 \mathrm{~cm}$ the curve became flatten; When the particle size of straw was $0.01 \mathrm{~cm}$, the oxygen index of compound material of straw was $25.8 \%$; When the particle size of straw was $1 \mathrm{~cm}$, the oxygen index of compound material of straw was $21.4 \%$; by calculation, flame retardant of the straw reduced 1.2 times. The results showed that the particle size of straw was smaller, the consequent of package was better. Therefore, the subsequent experiments used particle size of straw not more than $0.01 \mathrm{~cm}$.

\subsection{Mechanism of GO/straw flame Retardant}

The gradual change curve of oxygen index in straw composites with the change of GO is as follows fig.4, Straw particle is constant, when the content of GO increased, the oxygen index shows upward trend. The oxygen index of straw without GO is $19.6 \%$, yet the oxygen index of GO/straw composite reaches $29.4 \%$. According to GB8624-2012 "building materials and products of combustion performance classification", it reaches the national B level standard. 


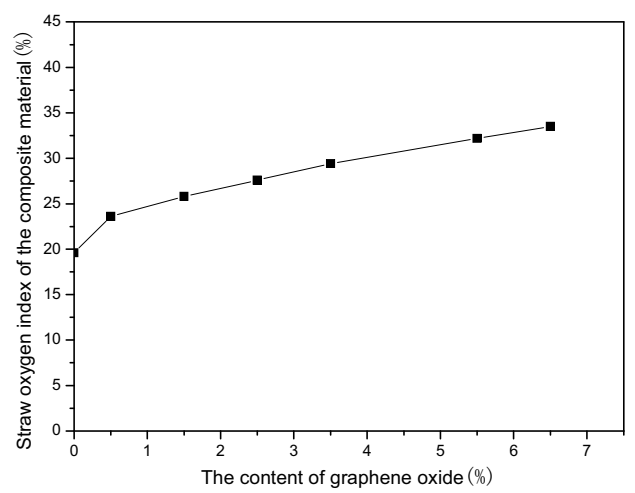

Fig.4 The gradual change curve of oxygen index in straw composites with the change of GO

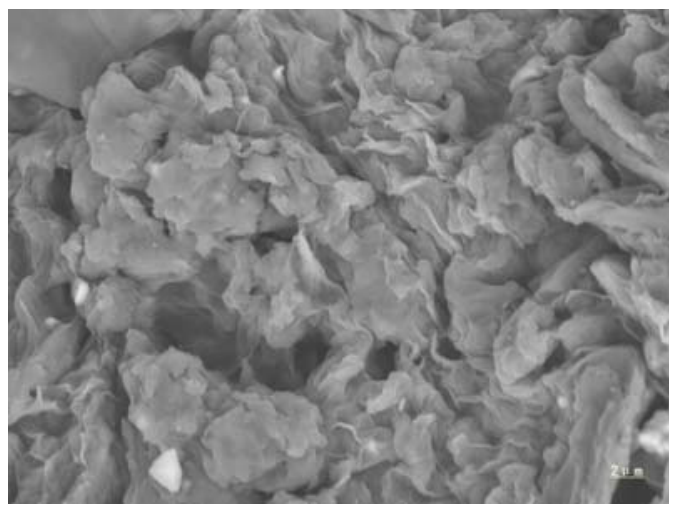

Fig.5 Composite section microscopic diagram

The density of GO increase with solution evaporation, the reaction center is straw paticles through the physical function at high speed stirring, which cause graphene oxide's encapsulated on straw surface. Because of the Van Edward force between the GO sheets, it forms a dense protective carbon layer on the surface of straw particle after drying. The "winding road" effect of GO film in the composite material can obstruct gas molecular penetration layer by layer, as follow from fig.5.there by increasing the gas barrier properties of composite materials. In another way, GO layer can be useful barrier of heat ${ }^{[4]}$, which increases flame retardant of the composite.

\section{Conclusion}

We made $\mathrm{GO} /$ straw flame retardant composite material through preparation of the spin-coating evaporation. With the increase of GO content, GO/straw oxygen index of the composite increased significantly, when the GO content is $3.5 \%, \mathrm{GO} /$ straw composites oxygen index was $29.4 \%$.According to GB 8624-2012 "building materials and products of combustion performance classification", the GO/straw composite has reached national B level standard. It can be applied in the field of straw board and insulation flame retardant materials. 


\section{Acknowledgement}

This work was financially supported by the Jilin province comprehensive utilization of straw technology innovation platform under special foundation.

\section{References}

1. Li-guang Xiao, Jing-hui Li, Bao-yu Zhou, Dong-lin Shao, Straw green energy materials research performance, J.Jilin Institute of Architectural Engineering, 6 (2008)1-6.

2. Da Kang, Wen-bin $\mathrm{Hu}$, Progress graphene composites, J.Journal of Inorganic Materials, 3(2013)236-246.

3. Park S, Ruoff RS, Chemical methods for the production of graphenes, Nat Nanotechnol,(2009)217-24.

4. Jing Hu, Xing-jian Li, Feng Zhang, Dao-xing Sun, Yi-heng Zhang, Pressurized airtight $\mathrm{GO} /$ aqueous polyurethane nanometer of composites preparation and flame retardant properties, J.Polymer Materials Science and Engineering, 3(2015)163-168. 